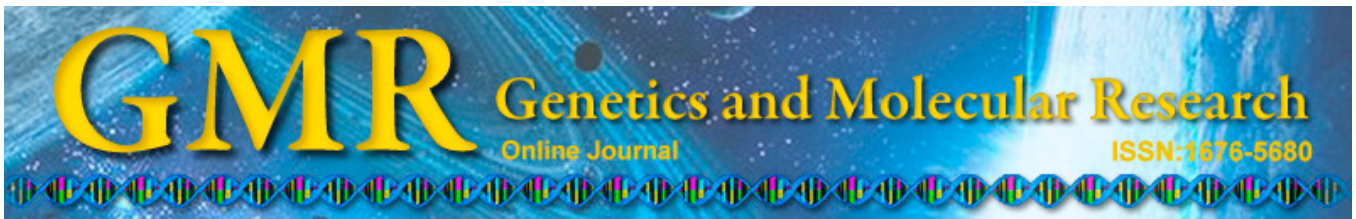

\title{
Zhi-Long-Huo-Xue-Tong-Yu modulates mitochondrial fission through the ROCK1 pathway in mitochondrial dysfunction caused by streptozotocin-induced diabetic kidney injury
}

W.H. Wu ${ }^{1 *}$, M.P. Zhang ${ }^{2 *}$, S.J. Yang ${ }^{3}$, Q. Liu ${ }^{1}$, X. Bai ${ }^{3}$ J.H. Qin B. Peng1, S.T. Ou ${ }^{1}$, J. Liu ${ }^{1}$, C.Y. Zhao ${ }^{2}$, Q. Zhang ${ }^{2}$, Q.D. Hu ${ }^{2}$ and L. Xue ${ }^{4}$

${ }^{1}$ Department of Nephropathy, Affiliated Hospital of Luzhou Medical College, Luzhou, Sichuan, China

${ }^{2}$ Department of Nephrology, Traditional Chinese Medical Hospital, Luzhou Medical College, Luzhou, Sichuan, China

${ }^{3}$ Department of Cardiology, Traditional Chinese Medical Hospital, Luzhou Medical College, Luzhou, Sichuan, China

${ }^{4}$ Department of Urology, Affiliated Hospital of Luzhou Medical College, Luzhou, Sichuan, China

*These authors contributed equally to this study.

Corresponding author: S.J. Yang

E-mail:whhhwei@126.com

Genet. Mol. Res. 14 (2): 4593-4606 (2015)

Received July 15, 2014

Accepted March 6, 2015

Published May 4, 2015

DOI http://dx.doi.org/10.4238/2015.May.4.18

ABSTRACT. Zhi-Long-Huo-Xue-Tong-Yu (ZLHXTY) is a defined mixture of 5 herbs developed by Professor S.J. Yang according to the Buyang Huanwu decoction method, which has been recorded in the Yilingaicuo. This study investigated the renoprotective effects of ZLHXTY on mitochondrial dysfunction induced by diabetic kidney 
injury in a diabetic rat model. Diabetes was induced by a single intravenous injection of streptozotocin. Rats were daily fed either ZLHXTY or vehicle beginning in the 1st week after injection. Levels of mitofusin 2 ( $\mathrm{mfn} 2)$, dynamin-related protein 1 (Drp1), caspase-9, and rho-associated, coiled-coil-containing protein kinase 1 (ROCK1) were detected using Western blotting. Levels of intracellular calcium and adenosine triphosphate (ATP) were examined using an enzyme-linked immunosorbent assay. An electron microscopic examination of kidney tissue was performed. The levels of $\mathrm{mfn} 2$ and ATP in the diabetes and ZLHXTY groups decreased from the 4th week after modeling. The expression levels of Drp1, ROCK1, and caspase-9 increased in the diabetes group but decreased in the ZLHXTY group from the 4th week after modeling. Compared with the diabetes group, ZLHXTY treatment decreased the mesangial expansion index and proteinuria levels, and improved the pathological changes typical of diabetic kidney injury. Furthermore, ZLHXTY treatment inhibited the activation of ROCK1 and expression of Drp1 and caspase-9, but did not affect the expression of mfn2. This study indicates that ZLHXTY treatment could protect kidney tissue from diabetic injury through the ROCK1 pathway response to mitochondrial dysfunction induced by diabetes.

Key words: Zhi-Long-Huo-Xue-Tong-Yu; Mitochondrial dysfunction; Diabetic kidney injury

\section{INTRODUCTION}

Diabetic nephropathy, the most common complication of diabetes, is the largest cause of end-stage renal disease in developed countries. It is characterized by glomerulosclerosis and loss of glomerular capillaries that is associated with increased albuminuria, persistent proteinuria, and a decline in glomerular filtration rate. Although the mechanisms of diabetic nephropathy are not entirely clear, mitochondrial dysfunction (Sourris et al., 2012) has been proposed as an underlying cellular mechanism for renal injury induced by diabetes. It is generally accepted that mitochondria in mammals are organized into tubular networks that undergo shape change regulated by the balance of mitochondrial fusion and fission (Huang et al., 2011). Mitochondrial fusion is the physical merging of the outer and inner mitochondrial membranes of two originally distinct mitochondria (Otera and Mihara, 2011). Excessive mitochondrial fission (fragmentation) is necessary for cytochrome $\mathrm{c}$ release and the subsequent caspase activation, which is essential for intrinsic apoptosis (Thomas and Jacobson, 2012). An imbalance between mitochondrial fission and fusion may be responsible for diabetic nephropathy, according to previous studies (Tang et al., 2012; Wang et al., 2012).

Mitofusin $2(\mathrm{mfn} 2)$ is an essential transmembrane GTPase embedded in the mitochondrial outer membrane, and localized on the endoplasmic reticulum, mediating mitochondrial fusion (Tang et al., 2012). On the other hand, dynamin-related protein 1 (DLP1/Drp1) can regulate mitochondrial fission, possibly via interaction with the mitochondrial surface (Huang et al., 2011). Furthermore, rho-associated, coiled-coil-containing protein kinase 1 (ROCK1) is an upstream regulatory factor of mitochondrial dysfunction between mitochondrial fission 
and fusion (Wang et al., 2012). According to Wang's study, ROCK1, which is activated when bound to the GTP-bound form of rho (Huang et al., 2011), triggers mitochondrial fission via increased levels of Drp1 in diabetic nephropathy (Wang et al., 2012) and may induce more significant diabetic kidney damage.

Increasing evidence shows that traditional Chinese herbs could protect mitochondria from injury under a variety of pathological conditions (Zhao et al., 2008; Qiu et al., 2012; Yu et al., 2012). Herbs have been used as diabetic nephropathy treatments in traditional Chinese medicine (TCM) for centuries. For example, it was reported that the use of cinnamon, a major ingredient of GuiZhi, has a beneficial effect on glycemic control (Akilen et al., 2012). Similarly, astragaloside prevents glucose-induced podocyte apoptosis partly by restoring the balance of Bax and Bcl-2 expression and inhibiting caspase-3 activation (Gui et al., 2012). The ZhiLong-Huo-Xue-Tong-Yu (ZLHXTY) formula, developed by Professor S.J. Yang according to the Buyang Huanwu decoction method and recorded in the Yilingaicuo (Liu et al., 2011; Sihn, 2012), consists of a defined mixture of 5 herbs namely, ShuiZhi (Whitmaniapigra Whitman), Di Long [Pheretima aspergillum (E. Perrier)], Huang Qi (Astragalus membranaceus Fisch.), GuiZhi (Cinnamomum cassia Presl.), and Da XueTeng (Sargento doxacuneata [Oliv.] Rehd. et Wils.) It is associated with renoprotective effects in patients and was considered a treatment for diabetic nephropathy by our group.

To our knowledge, the renoprotective role of natural herbs in regulating the balance between mitochondrial fission and fusion has not been investigated. Meanwhile, the cell-signaling pathway by which ZLHXTY mediates its effect remains uncertain. Considering that natural herbs in TCM protect mitochondria from pathological injury, we questioned whether the renoprotective role of ZLHXTY depends on its interaction with the mitochondrial fission and fusion pathway. To explore this question, we administered ZLHXTY to a streptozotocin (STZ)-induced diabetic rat model. We investigated its renoprotective effects on the balance of mitochondrial fission/fusion and the ROCK1 pathway. Furthermore, the corresponding clinical manifestations and pathological kidney changes were evaluated.

\section{MATERIAL AND METHODS}

\section{Animals}

Male Sprague-Dawley rats were purchased from the animal centerin LuzhouMedical College. Animals weighing 220-250 g at the outset of the study were used. They were fed standard rat chow and housed at a temperature of $22^{\circ} \pm 0.5^{\circ} \mathrm{C}$ with 24 -h alternate light-dark cycles. The rats $(\mathrm{N}=54)$ were randomly divided into 3 groups: the diabetes, control, and ZLHXTY group. The study was performed according to the National Institute of Health (NIH) Guide for the Care and Use of Laboratory Animals and approved by the institutional animal Ethics Committee of Luzhou Medical College.

\section{Composition of ZLHXTY and preparation of the herbal extract}

The ZLHXTY formula consists of a defined mixture of 5 herbs, as mentioned above. This mixture was granted a patent by the State Intellectual Property Office of the People's Republic of China (No. 200810147774.1). The herbs were processed and extracted with aqueous acetone at the Pharmaceutical Department, Luzhou Medical College of Traditional Chinese 
Medicine Hospital under Good Manufacturing Practice (GMP) conditions. Each extract was monitored for the absence of contaminants (heavy metals, pesticides, and mycotoxins) prior to formulation. Our previously published reports (in China) provide information on the detailed composition of ZLHXTY, the Chinese, botanical and family names of each component plant, pharmacopoeia used for reference, clinical dosages of each component plant, high-performance liquid chromatography (HPLC) peak descriptions (Chen et al., 2009) for ZLHXTY, weight and yield of the product, and details about voucher samples and pharmacopoeia. The formulated ZLHXTY was subjected to HPLC fingerprinting analysis in which the major peak was identified as Astragalus; see Figure 1. The long-term and acute toxicological studies have been published previously (Luo et al., 2009; Bai et al., 2010).
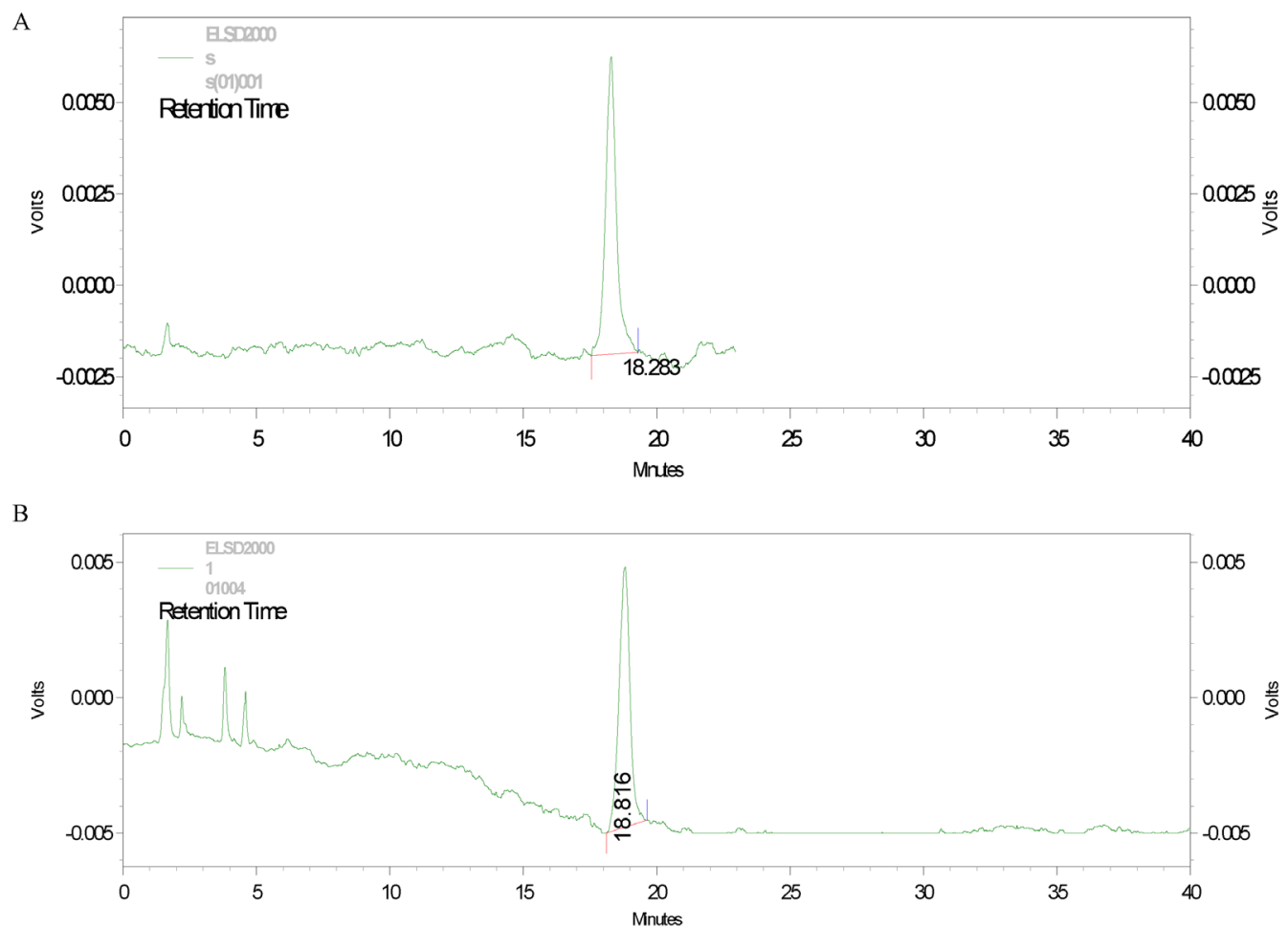

Figure 1. High-performance liquid chromatography (HPLC) fingerprinting analysis for the quality control and Zhi-Long-Huo-Xue-Tong-Yu (ZLHXTY) samples: the HPLC fingerprinting analysis was performed under the following conditions: mobile phase: acetonitrile-water $(32: 68)$; column temperature: $30^{\circ} \mathrm{C}$; flow rate: $1.0 \mathrm{~mL} / \mathrm{L}$; drift tube temperature: $105^{\circ} \mathrm{C}$; carrier gas flow rate: $2.7 \mathrm{~L} / \mathrm{min}$. An astragaloside IV sample (A) was used for quality control data; the major peak of ZLHXTY was identified as astragaloside IV (B).

\section{Protocol for causing STZ-induced diabetes in rats and ZLHXTY administration}

At the end of the 4th, 8th, and 12th week after modeling, 6 rats were sacrificed randomly from each group. Diabetes was induced by a single intravenous injection of STZ, Sigma; $50 \mathrm{mg} / \mathrm{kg}$ ) dissolved in citrate buffer with $\mathrm{pH}$ 4.2-4.5. Diabetes was defined as plasma glucose exceeding $16.7 \mathrm{mM}$ (Wu et al., 2011) according to our previous study. The control 
group rats were injected with citrate buffer only. Rats from the ZLHXTY group were fed ZLHXTY $(1.6 \mathrm{~g} / \mathrm{kg})$ by gavage daily beginning in the 1 st week after modeling. Concurrently, rats from the diabetes and control groups were fed an equal volume of saline.

\section{Protocol for the histological examination of kidney tissue}

Kidney tissue samples were isolated and embedded in paraffin to prepare $4-\mu \mathrm{m}$ tissue slices that were investigated using hematoxylin and eosin staining. A mesangial expansion index (MEI) was assigned, scoring in four levels from 0 to 3 , with the index scores defined as follows: 0 , normal glomeruli; 1 , matrix expansion occurred in up to $50 \%$ of a glomerulus; 2 , matrix expansion occurred in 50 to $75 \%$ of a glomerulus; 3 , matrix expansion occurred in 75 to $100 \%$ of a glomerulus. Scores were assigned for at least 30 glomeruli from kidney slices from each sample, and the means were calculated. Each slide was scored by a pathologist who was unaware of the experimental details (Lin et al., 2012).

\section{Measurement of mitochondrial-related protein and ROCK1 levels using Western blotting}

Using the Western blotting method as previously described (Wu et al., 2011), mfn2, Drp1, and ROCK1, which are considered upstream-regulatory factors of mitochondrial homeostasis, were examined. We used monoclonal mfn2 antibody (Abcam, UK, 1:500), monoclonal Drp1 antibody (Abcam, 1:500), rabbit monoclonal antibody to caspase-9 (Abcam, 1:500), and rabbit polyclonal antibody to ROCK1 (Abcam, 1:500). Tissues were homogenized in a radio-immunoprecipitation assay-lysis buffer containing $50 \mathrm{mM}$ Tris- $\mathrm{HCl}, \mathrm{pH} \mathrm{7.4,} 150$ $\mathrm{mM} \mathrm{NaCl}, 1 \% \mathrm{NP}-40,0.1 \%$ sodium dodecyl sulfate (SDS). Protein concentration was determined using the Bradford method and samples adjusted to equal concentrations. Samples were diluted with an equal volume of loading buffer and heated at $90^{\circ} \mathrm{C}$ for $5 \mathrm{~min}$ before being run on SDS-polyacrylamide gels. Electrophoresis was carried out in a Tris/glycine/SDS running buffer ( $25 \mathrm{mM}$ Tris, $0.25 \mathrm{M}$ glycin, $0.1 \% \mathrm{SDS}$ ). Following separation by electrophoresis, the proteins were transferred to polyvinylidenedifluoride membrane (Millipore, USA). After transfer, the membranes were blocked in 5\% non-fat milk in Tris-buffered saline $(\mathrm{pH} 7.4)$ supplemented with Tween 20 (TBST; $0.05 \%, \mathrm{v} / \mathrm{v}$ ) for $1 \mathrm{~h}$, before being probed with the following anti-rat antibodies in blocking solution overnight. Membranes were washed 3 times in TBST and then probed with peroxidase-conjugated goat anti-rabbit IgG (1:5000; Nan Jing, Jin Site Biotechnology Co., China) for $1 \mathrm{~h}$ at $37^{\circ} \mathrm{C}$. After washing, the specific signals were detected by enhanced chemiluminescence (Shinegene, Shanghai, Biotechnology Co., China).

\section{Examination of intracellular calcium levels in the renal tissue}

After the renal tissue was preserved with $0.9 \%$ saline, rat kidney cells were isolated from those samples as soon as possible. In brief, the renal cortical tissue was minced through a metallic mesh strainer and immediately placed in an ice-cooled buffer solution $\left(\mathrm{NaCl} 113 \mathrm{mM} ; \mathrm{KCl} 4 \mathrm{mM} ; \mathrm{NaHCO}_{3} 27.2 \mathrm{mM} ; \mathrm{KH}_{2} \mathrm{PO}_{4} 1 \mathrm{mM} ; \mathrm{MgCl}_{2} 1.2 \mathrm{mM} ; \mathrm{CaCl}_{2} 1\right.$ $\mathrm{mM}$; 4-(2-hydroxyethyl)-1-piperazineethanesulfonic acid (HEPES) $10 \mathrm{mM}$; Ca lactate 0.5 $\mathrm{mM}$; glucose $17.5 \mathrm{mM}$, glutamine $2 \mathrm{mM}$, and streptomycin $50 \mathrm{U} / \mathrm{mL}$; osmolality $298 \pm 2$ mOsm; $\mathrm{pH} 7.4)$, followed by an incubation with collagenase $(0.05 \% \mathrm{w} / \mathrm{v}$; Sigma-Aldrich) 
at $37^{\circ} \mathrm{C}$ for $60 \mathrm{~min}$, during which the buffer was equilibrated with $95 \% \mathrm{O}_{2} / 5 \% \mathrm{CO}_{2}$. Subsequently, an equal volume $(20 \mu \mathrm{L})$ of cell suspension was added to a $96-w e l l$ ELISA plate (Active Motif). There were 3 wells for each sample. After incubation with Fluo-3 AM (Beyotime, China) for $1 \mathrm{~h}$, the cells were washed twice with phosphate-buffered saline. The intracellular calcium level is reported as the fold-increase over a control value calculated using a microplate reader.

\section{Examination of the ATP level in the renal tissue}

ATP production was monitored by glucose-6-phosphate formation. Briefly, tissues were extracted with perchloric acid $(6 \%)$ and centrifuged $(8000 \mathrm{~g}$ for $10 \mathrm{~min})$. Subsequently, the extract was neutralized with $\mathrm{K}_{2} \mathrm{CO}_{3}(5 \mathrm{M})$ to $\mathrm{pH}$ 7. $\mathrm{NADP}^{+}(0.5 \mathrm{mM})$ and glucose 6-phosphate dehydrogenase $(0.25 \mathrm{U})$ were then added and ATP production was monitored from the NADPH content detected by spectrophotometry at $340 \mathrm{~nm}$. ATP level is reported as the foldincrease over a control value calculated using a microplate reader.

\section{Electron microscopy}

The fractions of kidney tissue were fixed in $2 \%$ glutaraldehyde in $0.1 \mathrm{M}$ sodium cacodylate, $\mathrm{pH} 7.4,0.1 \mathrm{M}$ sucrose, and $3 \mathrm{mM} \mathrm{CaCl}_{2}$, at $4^{\circ} \mathrm{C}$ overnight, and centrifuged to a pellet. The pellet was rinsed in $0.15 \mathrm{M}$ sodium cacodylate buffer containing $3 \mathrm{mM} \mathrm{CaCl}_{2}, \mathrm{pH} 7.4$, followed by post-fixation in $2 \%$ osmium tetroxide in $0.07 \mathrm{M}$ sodium cacodylate buffer containing $1.5 \mathrm{mM} \mathrm{CaCl}, \mathrm{pH} 7.4$ at $4^{\circ} \mathrm{C}$ for $2 \mathrm{~h}$, dehydrated in ethanol followed by acetone, and embedded in LX-112 (Ladd). Sections were contrasted with uranyl acetate followed by lead citrate and examined in a Leo 906 transmission electron microscope at $80 \mathrm{kV}$. Digital images were taken using a Morada digital camera.

\section{Statistical analysis}

Multiple-data sets between groups were analyzed using the Mann-Whitney test or the Kruskal-Wallis test followed by the Dunn post hoc test. Unless otherwise stated, $\mathrm{P}<0.05$ was considered to be statistically significant. Statistical analyses were performed using the PSS 17.0 software. Descriptive statistics are reported as means \pm standard error of the mean.

\section{RESULTS}

\section{Clinical characteristics of STZ-induced diabetic rats}

There were no baseline differences among the diabetes, control, and ZLHXTY groups. At the end of the 3rd day after modeling, the plasma glucose of rats that received the STZ injection significantly increased ( 3 times more than baseline). At the end of the 4 th week, the 24-h urine protein levels in the ZLHXTY and diabetes groups significantly increased; the difference between the 2 groups was statistically significant $(P<0.05)$. The levels of creatinine in the ZLHXTY and diabetes groups were higher than those in the control group after the 8th week $(\mathrm{P}<0.05)$. The creatinine levels in the ZLHXTY group were lower than those in the diabetes group $(\mathrm{P}<0.05)$; see Figure 2 . 


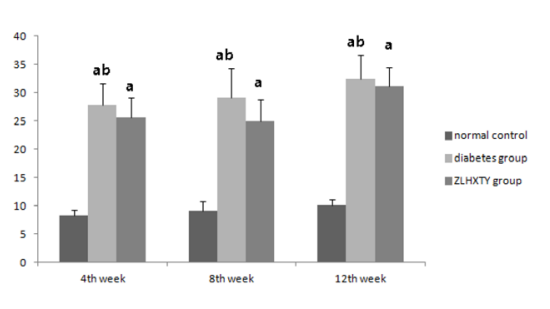

A

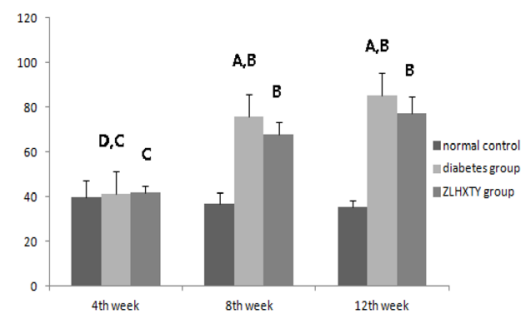

B

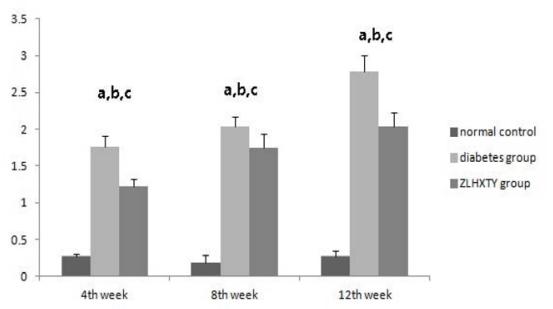

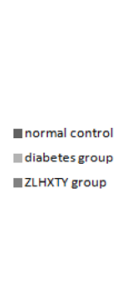

@normal control
\diabetes group
@ZLHXTY group
C

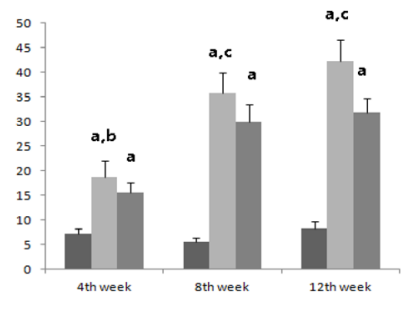

D

Figure 2. A. Glucose levels of the experimental rats (in $\mathrm{mM}$ ): All rats in both the diabetes and Zhi-Long-HuoXue-Tong-Yu (ZLHXTY) groups were administered a single intravenous injection of streptozotocin (Sigma; 50 $\mathrm{mg} / \mathrm{kg}$ ). The rats in the normal control group were administered an injection with an equal volume of saline; ${ }^{\text {a }} \mathrm{P}<$ 0.05 versus normal control group; ${ }^{b} \mathrm{P}>0.05$ versus diabetes group. B. Serum creatinine $(\mathrm{Cr})$ level of the rats (in $\mathrm{mM}$ ): There was no significant difference in the levels of serum creatinine at the end of the 4th week after modeling (normal control group $39.84 \pm 7.51 \mathrm{mM}$; diabetes group $41.24 \pm 4.32 \mathrm{mM}$; and ZLHXTY group $42.05 \pm 3.01 \mathrm{mM}$, $\mathrm{P}>0.05)$. The level of $\mathrm{Cr}$ increased in both the diabetes and ZLHXTY groups from the end of the 8th week after modeling. There was a significant difference between the diabetes and ZLHXTY groups at each time point. ${ }^{\mathrm{A}} \mathrm{P}<$ 0.05 versus normal control; ${ }^{\mathrm{B} P}<0.05$ versus ZLHXTY group; ${ }^{\mathrm{CP}}>0.05$ versus normal control; ${ }^{\mathrm{D} P}>0.05$ versus ZLHXTY group. C. 24-h urine protein level for rats used in our study $(\mathrm{mg} / 24 \mathrm{~h})$ : The 24-h urine protein levels in both the diabetes and ZLHXTY groups significantly increased after the end of 4th week. Except at the end of the 4th week after modeling (ZLHXTY group $15.51 \pm 2.21 / 24$ versus $18.75 \pm 3.31 \mathrm{mg} / 24 \mathrm{~h}, \mathrm{P}>0.05$ ), the 24-h urine protein level in the ZLHXTY group was lower than that in the diabetes group at each time point $(\mathrm{P}<0.05)$; ${ }^{\text {a }}<$ 0.05 versus normal control; ${ }^{b} \mathrm{P}<0.05$ : versus ZLHXTY group; ${ }^{\mathrm{P}}<0.05$ : versus ZLHXTY group. D. Mesangial expansion index (MEI): The levels of MEI in the diabetes and ZLHXTY groups were significantly higher than those in the normal control (diabetes group: $1.761 \pm 0.144$ at the end of 4th week, $2.034 \pm 0.138$ at the end of 8th week, and $2.785 \pm 0.212$ at the end of the 12th week; ZLHXTY group: $1.219 \pm 0.103$ at the end of 4th week, 1.751 \pm 0.182 at the end of 8 th week, and $2.032 \pm 0.201$ at the end of the 12 th week, $\mathrm{P}<0.05$ ). Meanwhile the level of MEI in the ZLHXTY group was lower than that in the diabetes group at each point $(\mathrm{P}<0.05)$; ${ }^{\text {aP }}<0.05$ versus normal control at each point. ${ }^{b} \mathrm{P}<0.05$ for the different points in the diabetes group. ${ }^{\mathrm{c}} \mathrm{P}<0.05$ versus the ZLHXTY group for each time point.

\section{Histological examination of kidney tissue in STZ-induced diabetic rats}

At the end of the 4th week after modeling, the rats in the diabetes and ZLHXTY groups showed glomerular mesangial expansion and basement membrane thickening, which was not observed in the normal control group. The levels of MEI in these 2 groups significantly increased at the end of the 4th week, 8th week, and 12th week. Between the 2 groups, the levels of MEI in the diabetes group were higher than those in the ZLHXTY group $(\mathrm{P}<0.05)$; see Figure 3. 


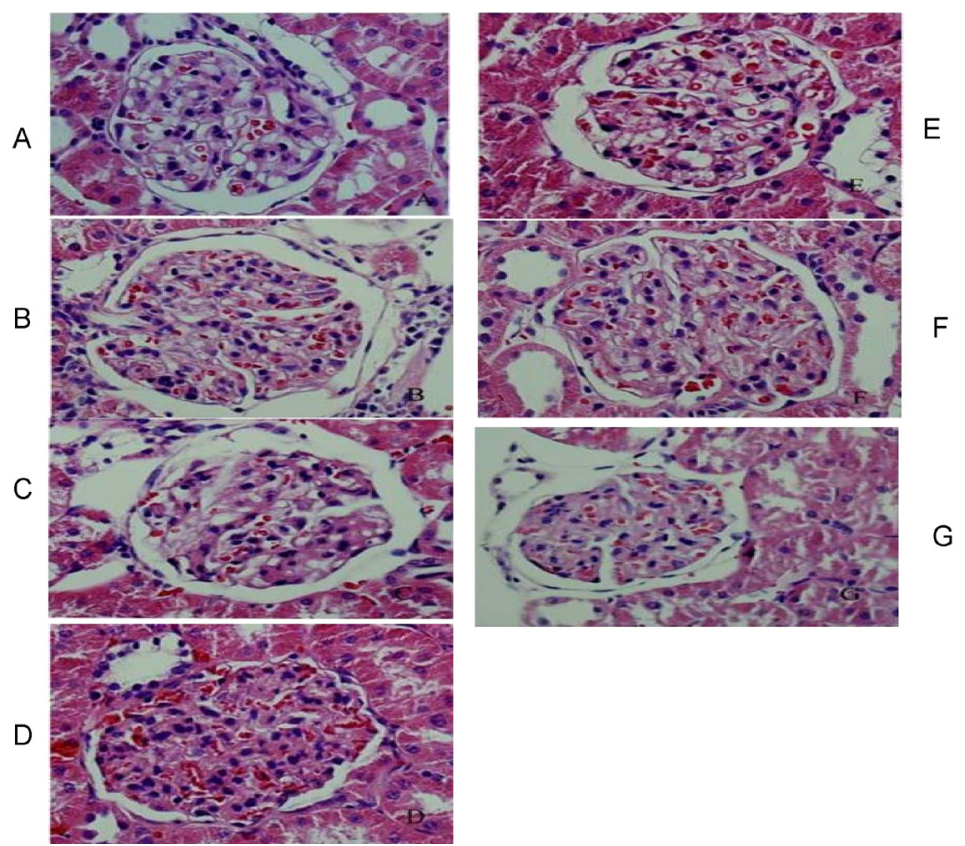

Figure 3. Histological examination of kidney tissue in streptozotocin (STZ)-induced rats: Diabetes was induced by a single STZ injection (Sigma: $50 \mathrm{mg} / \mathrm{kg}$ ) dissolved in citrate buffer with $\mathrm{pH}$ 4.2-4.5. Changes of glomerular pathology before the end of 12 th week were observed by light microscope (stained with hematoxylin and eosin). Compared with the normal control (A), the diabetes and Zhi-Long-Huo-Xue-Tong-Yu (ZLHXTY) groups developed some changes such as significantly increased red dye material, mesangial expansion, glomerular morphology fullness, and volume expansion, whereas the ZLHXTY group revealed only slight changes in the pathologies mentioned above. A. Normal control; B. diabetes group at the end of the 4th week; C. diabetes group at the end of the 8th week; D. diabetes group at the end of the 12th week; E. ZLHXTY group at the end of the 4th week; F. ZLHXTY group at the end of the 8th week; G. ZLHXTY group at the end of the 12th week.

\section{Change of mitochondrial-related protein levels in kidney tissue in STZ-induced rats and those treated with ZLHXTY}

Drp1 and mfn2 were considered mitochondrial-related proteins in our study, through which mitochondrial dysfunction between mitochondrial fission and fusion was examined. It was shown through Western blotting that, compared with the normal control group, that the Drp1 and caspase-9 levels in the diabetes and ZLHXTY groups significantly increased from the end of 4th week after modeling. Meanwhile, the Drp1 and caspase-9 levels in the ZLHXTY group were lower than those in the diabetes group at the same point $(\mathrm{P}<0.05)$. On the other hand, there was a significant difference in $\mathrm{mfn} 2$ levels between these 2 groups and the normal control from the end of 4th week after modeling $(\mathrm{P}<0.05)$. Meanwhile, there was no significant difference in $\mathrm{mfn} 2$ levels between the ZLHXTY and diabetes groups $(\mathrm{P}>0.05)$. With respect to the hypothesis that ROCK1 is an upstream-regulatory factor of mitochondrial dysfunction between mitochondrial fission and fusion, our data showed that the level of ROCK1 increased in the ZLHXTY and diabetes groups from the 4th week after modeling and peaked at the end of the 12th week $(\mathrm{P}<0.05)$. Among these, the level of ROCK1 in the ZLHXTY group was lower than that in the diabetes group at each point; see Figure 4. 

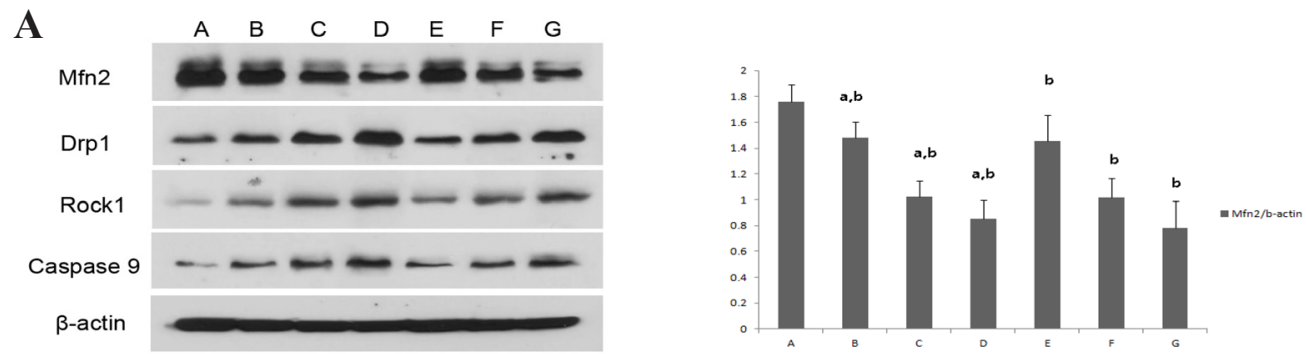

B

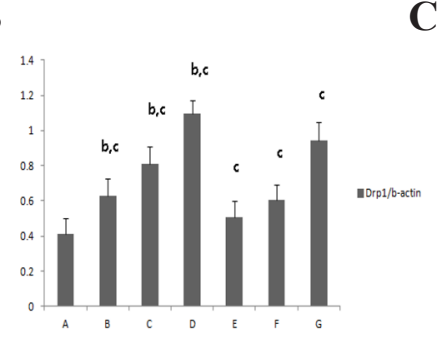

C

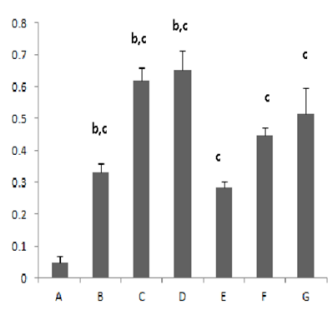

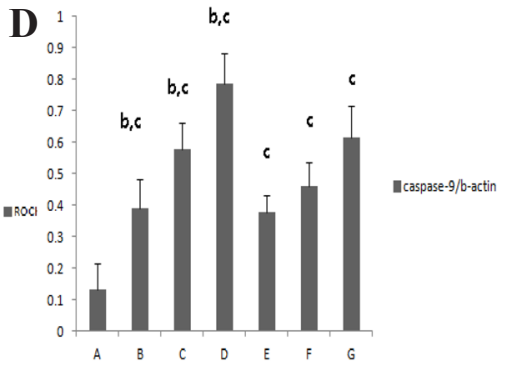

Figure 4. A. Level of mitofusin $2(\mathrm{mfn} 2)$ shown by Western blotting. Compared to the normal control $(1.758 \pm$ $0.132)$, the level of $\mathrm{mfn} 2$ in both the diabetes group $(1.477 \pm 0.124$ at the end of the 4 th week, $1.026 \pm 0.118$ at the end of the 8th week, and $0.854 \pm 0.143$ at the end of the 12th week) and Zhi-Long-Huo-Xue-Tong-Yu (ZLHXTY) group $(1.453 \pm 0.201$ at the end of the 4th week, $1.014 \pm 0.154$ at the end of the 8th week, and $0.782 \pm 0.208$ at the end of the 12th week) decreased from the end of the 4th week after modeling and was at its lowest at the end of the 12th week. However, there was no significant difference between the ZLHXTY and diabetes groups from the end of the 4th week after modeling. A: normal control; B: diabetic renal tissue at the end of the 4th week; C: diabetic renal tissue at the end of the 8th week; D: diabetic renal tissue at the end of the 12th week; E: ZLHXTY group at the end of the 4th week; F: ZLHXTY group at the end of the 8th week; G: ZLHXTY group at the end of the 12th week; ${ }^{\text {P P }>}$ 0.05 versus ZLHXTY group; ${ }^{b} \mathrm{P}<0.05$ versus normal control. B. Level of dynamin-related protein 1 (Drp1) shown by Western blotting. Compared to the normal control $(0.413 \pm 0.085$ in normal control), the level of Drp1 in both the diabetes group $(0.627 \pm 0.102$ at the end of the 4th week, $0.812 \pm 0.094$ at the end of the 8th week, and 1.096 \pm 0.073 at the end of the 12th week) and ZLHXTY group $(0.506 \pm 0.091$ at the end of the 4 th week, $0.602 \pm 0.089$ at the end of the 8th week, and $0.942 \pm 0.105$ at the end of the 12th week) increased from the end of 4th week after modeling and peaked at the end of the 12th week. Meanwhile, the level of Drp1 in the ZLHXTY group was higher than that in the diabetes group at each point. ${ }^{b} \mathrm{P}<0.05$ versus ZLHXTY group; ${ }^{\mathrm{c}} \mathrm{P}<0.05$ versus normal control. $\mathbf{C}$. Level of rho-associated, coiled-coil-containing protein kinase 1 (ROCK1) shown by Western blotting. Compared to the normal control $(0.05 \pm 0.02$ in normal control $)$, the level of ROCK 1 in both the diabetes group $(0.332 \pm 0.028$ at the end of the 4th week, $0.619 \pm 0.041$ at the end of the 8th week, and $0.654 \pm 0.058$ at the end of the 12th week) and ZLHXTY group $(0.284 \pm 0.019$ at the end of the 4th week, $0.450 \pm 0.023$ at the end of the 8 th week, and 0.515 \pm 0.082 at the end of the 12th week) increased from the end of the 4th week after modeling and peaked at the end of the 12th week. Meanwhile, the level of ROCK1 in the ZLHXTY group was lower than that in the diabetes group at each point. ${ }^{b} \mathrm{P}<0.05$ versus ZLHXTY group; ${ }^{~} \mathrm{P}<0.05$ versus normal control. D. Level of caspase- 9 shown by Western blotting. Compared to the normal control ( $0.132 \pm 0.082$ in normal control), the level of caspase-9 in both the diabetes group $(0.391 \pm 0.093$ at the end of the 4th week, $0.577 \pm 0.084$ at the end of the 8th week, and 0.787 \pm 0.097 at the end of the 12th week) and ZLHXTY group $(0.376 \pm 0.054$ at the end of the 4 th week, $0.462 \pm 0.076$ at the end of the 8th week, and $0.616 \pm 0.099$ at the end of the 12th week) increased from the end of the 4th week after modeling and reached its lowest level at the end of the 12th week. Meanwhile, the level of caspase-9 in the ZLHXTY group was higher than that in the diabetes group at each point. ${ }^{b} \mathrm{P}<0.05$ versus ZLHXTY group; ${ }^{\mathrm{P}}<$ 0.05 versus normal control. 


\section{Change in ATP production in diabetic renal tissue}

It is known that mitochondria are an important source of ATP production in cells. ATP reduction may suggest that there could be mitochondrial dysfunction. Our data show that the ATP level in the diabetes and ZLHXTY groups decreased from the end of 4th week after modeling and reached its lowest point at the end of 12th week $(\mathrm{P}<0.05)$. Meanwhile, the ATP level in the ZLHXTY group was higher than that in the diabetes group at each point $(\mathrm{P}<0.05)$; see Figure $5 \mathrm{~A}$.

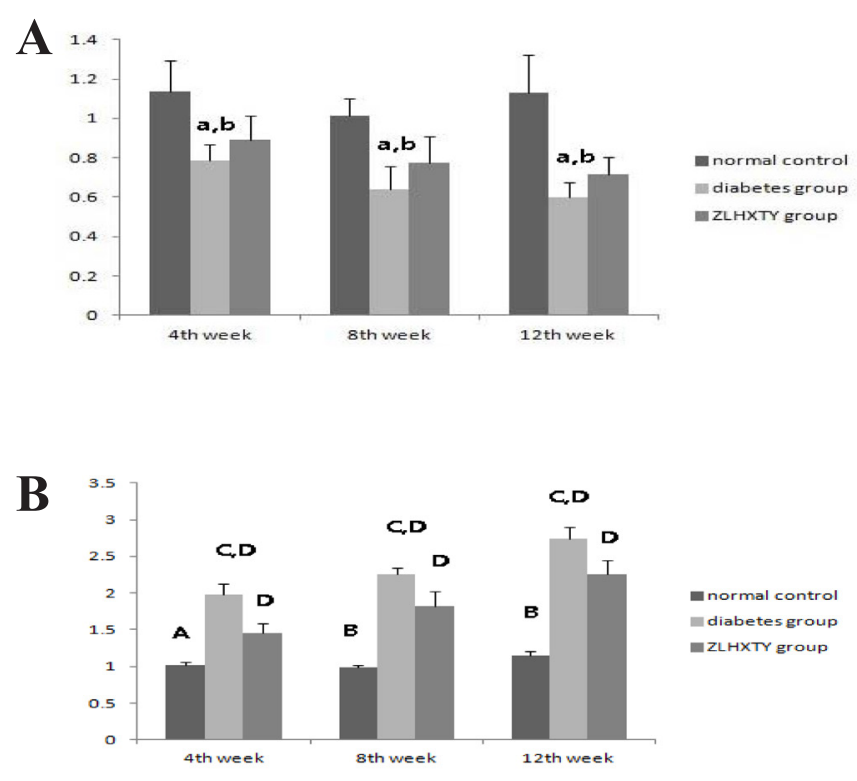

Figure 5. A. Level of adenosine triphosphate (ATP). The level of ATP in the normal control was $1.138 \pm 0.153$. The level of ATP in both the diabetes group $(0.784 \pm 0.084$ at the end of the 4 th week, $0.641 \pm 0.117$ at the end of the 8th week, and $0.597 \pm 0.076$ at the end of the 12th week) and Zhi-Long-Huo-Xue-Tong-Yu (ZLHXTY) group $(0.891 \pm 0.124$ at the end of the 4th week, $0.772 \pm 0.138$ at the end of the 8th week, and $0.715 \pm 0.091$ at the end of the 12th week) significantly decreased from the end of the 4 th week after modeling $(\mathrm{P}<0.05)$. Meanwhile the ATP level in the ZLHXTY group was significantly higher than that in the diabetes group at each point. ${ }^{a} \mathrm{P}<0.05$ versus normal control; ${ }^{b} \mathrm{P}<0.05$ versus ZLHXTY group at same point. B. Level of infracellular calcium. The level of intracellular calcium in the normal control group at baseline was $1.012 \pm 0.034$. The levels of intracellular calcium in the ZLHXTY group ( $1.458 \pm 0.132$ at the end of the 4th week, $1.183 \pm 0.215$ at the end of the 8th week, and 2.257 \pm 0.193 at the end of the 12th week) and diabetes group (1.985 \pm 0.147 at the end of the 4th week, $2.259 \pm 0.092$ at the end of the 8th week, and $2.742 \pm 0.156$ at the end of the 12th week) were significantly higher than those in the normal control in a time-dependent manner $(\mathrm{P}<0.05)$. The level of intracellular calcium in the diabetes group was higher than that in the ZLHXTY group at each point. A: the level of intracellular calcium in the 4th week after modeling in the normal control group was considered as baseline in this study; ${ }^{\mathrm{B} P}>0.05$ versus baseline; ${ }^{\mathrm{C}} \mathrm{P}<0.05$ versus ZLHXTY group at each point; ${ }^{\mathrm{D} P}<0.05$ among the points in the same group.

\section{Change in intracellular calcium levels due to ZLHXTY treatment and STZ-induc- tion of diabetes}

Intracellular $\mathrm{Ca}^{2+}$ uptake is thought to provide an important signal to increase energy production to meet demand; however, in excess, it can also trigger cell death, which indicates the level of mitochondrial function. In our study, it was shown that the levels of intracellular 
calcium significantly increased after modeling in the diabetes and ZLHXTY groups from the end of 4th week after modeling. Meanwhile, the levels of intracellular calcium in the ZLHXTY group were lower than those in the diabetes group at each time point; see Figure 5B.

\section{Ultrastructural changes due to ZLHXTY treatment and STZ-induction of diabetes}

In our study, to observe changes in the ultrastructure of mitochondria and the glomerular basement membrane, an electron microscopy test for renal tissue was conducted. Basement membrane thickening, glomerular podocyte fusion, and mitochondrial swelling were observed in the ZLHXTY and diabetes groups from the 4th week after modeling, which was rarely observed in the normal control group. Among the 2 groups, the kidney tissue injury in the diabetes group was significantly greater than that in the ZLHXTY group at the 4th week, 8th week, and 12th week; see Figure 6.

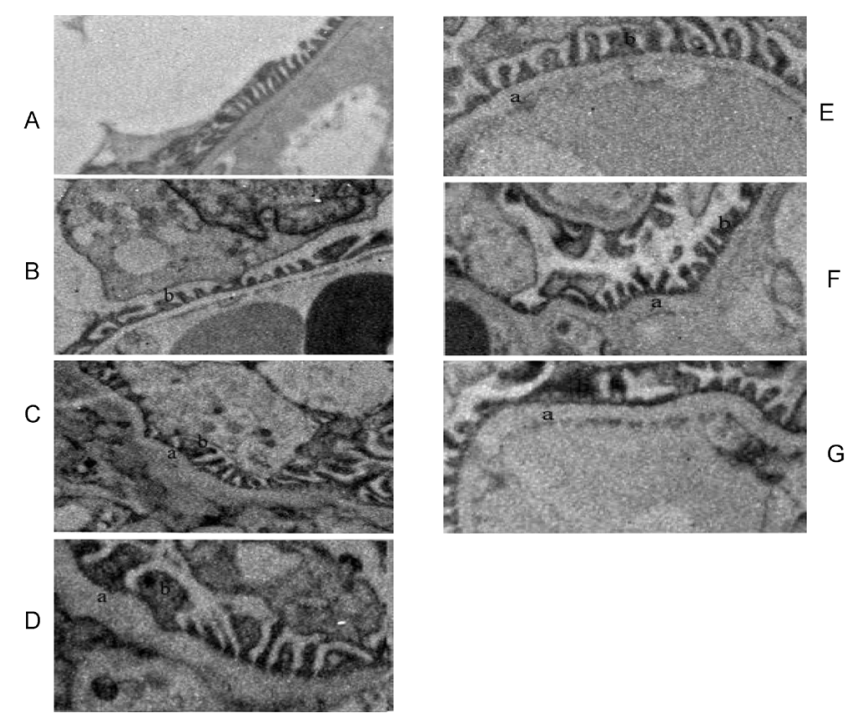

Figure 6. Glomerular ultramicropathological changes before the end of the 12th week after modeling. Basement membrane thickening (a) and foot process fusion (b) can be observed in the diabetes and Zhi-Long-Huo-Xue-Tong$\mathrm{Yu}$ (ZLHXTY) groups. The ZLHXTY group had relatively minor damage compared to that in the diabetes group. A. Normal control; B. diabetes group at the end of the 4th week; C. diabetes group at the end of the 8th week; D. diabetes group at the end of the 12th week; E. ZLHXTY group at the end of the 4th week; F. ZLHXTY group at the end of the 8th week; G. ZLHXTY group at the end of the 12th week.

\section{DISCUSSION}

This study showed that 1) ZLHXTY treatment $(1.6 \mathrm{~g} / \mathrm{kg})$ by gavage daily in STZ-induced diabetic rats reduced diabetic renal damage and protected renal function; 2) ZLHXTY treatment in vivo attenuated mitochondrial dysfunction induced by hyperglycemia in diabetic renal tissue.

ZLHXTY is a compound containing multiple ingredients, developed by Professor Yang Sijing according to the Buyang Huanwu decoction method (Liu et al., 2011) in the meridian theory of TCM, which is usually used to treat chronic vascular disorder, considered as wind-phlegm-stagnation syndrome according to TCM theory (Luo et al., 2010a; Liao et 
al., 2011; He et al., 2011). According to the Buyang Huanwu decoction method, in which Astragalus (Huang Qi) is a representative drug replenishing Qi, we used Astragalus (Huang Qi) in an amount accounting for $1 / 2$ of the entire prescription dosage of ZLHXTY. This explains the major peak in HPLC fingerprinting analysis.

According to our previous study, ZLHXTY protected cells from apoptosis after ischemia/reperfusion induced by acute cerebral infarction (Luo et al., 2010b). Therefore, apoptosis may be related to mitochondrial function in pathological conditions. Intrinsic apoptosis may be related to permeabilization of mitochondria (Suen et al., 2008; Thomas et al., 2012), but also the imbalance of mitochondrial fusion and fission may induce apoptosis. For example, excessive mitochondrial fission is essential for apoptosis (Thomas et al., 2012). Tang et al. (2012) showed that $\mathrm{mfn} 2$ overexpression attenuated pathological changes in the kidneys of diabetic rats. Meanwhile, Wang et al. (2012) showed that deletion of ROCK1 in diabetic mice prevented mitochondrial fission, whereas podocyte-specific cA-ROCK1 mice exhibited increased mitochondrial fission associated with less significant kidney damage, indicating that mitochondrial dysfunction plays an important role in diabetic nephropathy. Anti-mitochondrial dysfunction might be a potential therapeutic target for the treatment of diabetic nephropathy.

Chinese traditional medicines were used widely in treating the diabetic complication known as "Xiaoke" and significant evidence has been accumulated (Tong et al., 2012). Diabetic nephropathy is a common diabetic microvascular complication. In TCM theory, diabetic nephropathy is also considered as wind-phlegm-stagnation syndrome induced by Qi deficiency and congestion.

Clinical parameters in our study indicative of diabetic nephropathy were markedly ameliorated by ZLHXTY treatment. ZLHXTY treatment is associated with decreased kidney volume (as indicated by MEI) and reduced proteinuria in diabetic rats, which indicates that it slows the progression of diabetic nephropathy. ZLHXTY treatment also inhibited Drp1 and ROCK1, which are considered upstream regulatory factors of mitochondrial dysfunction between mitochondrial fission and fusion. Thus, we believe that the potential mechanism of the protective effect of ZLHXTY may be due to a protective role in mitochondria.

However, no significant differences were observed between the ZLHXTY and diabetic groups through Western blotting analysis for $\mathrm{mfn} 2$ at the end of the 12th week of establishing the diabetic state. This phenomenon can be explained by the following: 1) alteration of the Drp1 level is the major change following ZLHXTY treatment; ROCK1 promotes mitochondrial fission via Drp1 phosphorylation at Ser600 (Wang et al., 2012) and ZLHXTY's protective role may involve the ROCK1-Drp1 pathway, and 2) diabetic nephropathy is accompanied by increased generation of reactive oxygen species. Oxidative stress has emerged as a critical pathogenic process in the development of diabetic nephropathy (Yamagishi and Matsui, 2010). The correlation between imbalance in mitochondrial fission-fusion and oxidative stress has been examined. Wu at al. (2010) showed that mitochondrial oxidative stress mediated through Drp1 and $\mathrm{mfn} 2$ causes an imbalance in mitochondrial fission-fusion, which contributes to mitochondrial and cell dysfunction. In our study, the influence of high glucose on imbalance in mitochondrial fission-fusion may be explained by oxidative stress.

Although multiple pathways may be involved in regulating the imbalance in mitochondrial fission-fusion, ROCK1 has been considered as an upstream-regulatory factor of mitochondrial dysfunction between mitochondrial fission and fusion. Previous studies have confirmed the significant role of ROCK1 in diabetic nephropathy as follows: 1) unregulated ROCK1 in the diabetic nephropathy model may be associated with loss of megalin/cubilin 
and an increase in transforming growth factor- $\beta$, interleukin- $1 \beta$, and fibrosis in the kidney (Zhou et al., 2011); 2) ROCK1 activation could trigger mitochondrial fission via increased levels of Drp1 in diabetic nephropathy as mentioned above (Wang et al., 2012); 3) the role of ROCK1 in diabetic nephropathy may be through an advanced glycation end-products pathway (Reiniger et al., 2010). Among these, the evidence on the relationship between ROCK1 and $\mathrm{mfn} 2$ is very limited. Therefore, we hypothesize that ZLHXTY directly inhibits Drp1 through the ROCK1 pathway, further protecting cells from apoptosis. This mechanism might account for the protective role of ZLHXTY.

To our knowledge, the protective influence of Astragalus (Huang Qi) by inhibition of the ROCK1 pathway has not been reported. We believe this protective role to be a cumulative or synergistic effect of multiple compounds present in the herbal extract besides Astragalus that work via the ROCK1 pathway. For example, cinnamon oil is also considered a treatment for diabetes, as mentioned above. However, astragaloside data were considered as a quality control data for ZLHXTY. This quality control method can be explained by the following: 1) Astragalus (Huang Qi) is the main compound of ZLHXTY according to the Buyang Huanwu decoction method; 2) cinnamon oil and other compounds from ZLHXTY are unstable, thus difficult to use for quality control data.

The present study has some limitations. Firstly, although ZLHXTY treatment may attenuate mitochondrial dysfunction, there was no significant effect of ZLHXTY on $\mathrm{mfn} 2$ and mitochondrial fusion imbalance. Analysis of other TCM herbs may allow further extension of the present study. Secondly, tests (such as terminal deoxynucleotidyltransferased UTP nick end-labeling staining) to confirm a direct morphologic change associated with apoptosis have not been performed. Our data only focused on mitochondrial dysfunction, which may target apoptosis.

In summary, ZLHXTY attenuates many pathologic changes in kidney structure and function associated with diabetes in rats. It plays a renoprotective role, possibly by sustaining mitochondrial function through the ROCK1-Drp1 pathway.

\section{ACKNOWLEDGMENTS}

Research supported by the National Natural Science Foundation of China (\#81200533), the Science and Technology Bureau of Luzhoucity (\#128, 2010; \#108, 2011) and the Sichuan Provincial Health Department

\section{REFERENCES}

Akilen R, Tsiami A, Devendra D and Robinson N (2012). Cinnamon in glycaemic control: Systematic review and meta analysis. Clin. Nutr. 31: 609-615.

Bai X, Yang SJ, Luo M, et al. (2010). Long-term Toxicological Study on ZhilongHuoxueTongyu Capsule. J. Liaoning Univ. Trad. Chin. Med. 12: 34-37.

Chen YL, Zhao J, Pu QR, et al. (2009). Determination of Astragaloside ${ }^{\mathrm{IV}}$ in Zhilonghuoxuetongyu capsules. Progr. Mod. Biomed. 9: 4113-4115.

Gui D, Guo Y, Wang F, Liu W, et al. (2012). Astragaloside IV, a novel antioxidant, prevents glucose-induced podocyte apoptosis in vitro and in vivo. PLoS One 7: e39824.

He L, Wen T, Yan S, Li R, et al. (2011). Reevaluation of the effect of Dianxianning on seizure rate of refractory epilepsy as additive treatment in clinical practice. Front Med. 5: 229-234.

Huang P, Galloway CA and Yoon Y (2011). Control of mitochondrial morphology through differential interactions of mitochondrial fusion and fission proteins. PLoS One 6: e20655.

Liao HL, Yang SJ, Xi XL, et al. (2011). Intervention of ZhilongHuoxueTongyu Capsule on IL-6, TNF-a and CRP of Renal Hypertensive rats. Henan Trad. Chin. Med. 31: 1239-1241. 
Lin CC, Lin LT, Yen MH, Cheng JT, et al. (2012). Renal protective effect of xiao-chai-hu-tang on diabetic nephropathy of type 1-diabetic mice. Evid. Based Complement. Alternat. Med. 2012: 984024.

Liu Y, Lin R, Shi X, Fang Z, et al. (2011). The roles of buyanghuanwu decoction in anti-inflammation, antioxidation and regulation of lipid metabolism in rats with myocardial ischemia. Evid. Based Complement. Alternat. Med. 2011: 561396.

Luo G, Bai X and Yang SJ (2010a). Zhilonghuoxutongyu capsule to acute cerebral infarction patient hemotology target and blood vessel bast secretion function influence. JETCM. 19: 545-547.

Luo G, Bai X and Yang SJ (2010b). The effect of Zhi Long HuoXue Tong Yu capsule on the apoptosis level and apoptosis related gene expression in rats nerve cells after ischemia/reperfusion induced by acute cerebral infarction. Lishizhen Med. Mat. Med. Res. 21: 2691-2692.

Luo M, Yang SJ, Song YZ, et al. (2009). Acute toxicological study of Zhi-long HuoxueTongyu Capsule. Lishizhen Med. Mat. Med. Res. 20: 1939-1940.

Otera $\mathrm{H}$ and Mihara K (2011). Molecular mechanisms and physiologic functions of mitochondrial dynamics. J. Biochem. 149: 241-251.

Qiu W, Zhou Y, Jiang L, Fang L, et al. (2012). Genipin inhibits mitochondrial uncoupling protein 2 expression and ameliorates podocyte injury in diabetic mice. PLoS One 7: e41391.

Reiniger N, Lau K, McCalla D, Eby B, et al. (2010). Deletion of the receptor for advanced glycation end products reduces glomerulosclerosis and preserves renal function in the diabetic OVE26 mouse. Diabetes 59: 2043-2054.

Sihn KH (2012). The anatomical revolution and the transition of anatomical conception in late imperial China. Uisahak 21: $67-100$.

Sourris KC, Harcourt BE, Tang PH, Morley AL, et al. (2012). Ubiquinone (coenzyme Q10) prevents renal mitochondrial dysfunction in an experimental model of type 2 diabetes. Free Radic. Biol. Med. 52: 716-723.

Suen DF, Norris KL and Youle RJ (2008). Mitochondrial dynamics and apoptosis. Genes Dev. 22: 1577-1590.

Tang WX, Wu WH, Zeng XX, Bo H, et al. (2012). Early protective effect of mitofusion 2 overexpression in STZ-induced diabetic rat kidney. Endocrine 41: 236-247.

Thomas KJ and Jacobson MR (2012). Defects in mitochondrial fission protein dynamin-related protein 1 are linked to apoptotic resistance and autophagy in a lung cancer model. PLoS One 7: e45319.

Tong XL, Dong L, Chen L and Zhen Z (2012). Treatment of diabetes using traditional Chinese medicine: past, present and future. Am. J. Chin. Med. 40: 877-886.

Wang W, Wang Y, Long J, Wang J, et al. (2012). Mitochondrial fission triggered by hyperglycemia is mediated by ROCK1 activation in podocytes. Cell Metab. 15: 186-200.

Wu S, Zhou F, Zhang Z and Xing D (2010). Mitochondrial oxidative stress causes mitochondrial fragmentation via differential modulation of mitochondrial fission-fusion proteins. FEBS J. 278: 941-954.

Wu WH, Zhang MP, Zhang F, Liu F, et al. (2011). The role of programmed cell death in streptozotocin-induced early diabetic nephropathy. J. Endocrinol. Invest. 34: e296-301.

Yamagishi S and Matsui T (2010). Advanced glycation end products, oxidative stress and diabetic nephropathy. Oxid. Med. Cell Longev. 3: 101-108.

Yu Y, Wang S, Nie B, Sun Y, et al. (2012). Effect of Astragali radix injection on myocardial cell mitochondrial structure and function in process of reversing myocardial cell hypertrophy. ZhongguoZhong Yao ZaZhi 37: 979-984.

Zhao HW, Qin F, Liu YX, Huang X, et al. (2008). Antiapoptotic mechanisms of Chinese medicine formula, Guan-Xin-ErHao, in the rat ischemic heart. Tohoku J. Exp. Med. 216: 309-316.

Zhou L, Liu F, Huang XR, Liu F, et al. (2011). Amelioration of albuminuria in ROCK1 knockout mice with streptozotocininduced diabetic kidney disease. Am. J. Nephrol. 34: 468-475. 